\title{
Distal Anterior Cerebral Artery Mirror Aneurysms and Middle Cerebral Artery Aneurysms
}

\author{
-Case Report- \\ Cem Dinc, Ahmet Celal IPlikcioglu, Kerem BiKMAZ, \\ Serkan KOSDERE, and Yüksel NAVRUZ \\ Neurosurgery Clinic, Ministry of Health Okmeydani Education and Research Hospital, \\ Istanbul, Turkey
}

\begin{abstract}
A 52-year-old woman presented with bilateral distal anterior cerebral artery (ACA) mirror aneurysms, in addition to two right middle cerebral artery (MCA) aneurysms. The left distal ACA and right MCA aneurysms were clipped through right interhemispheric and right pterional craniotomies. The right distal ACA aneurysm was thrombosed. Distal ACA aneurysms tend to be multiple, but mirror aneurysms are rare, especially in the absence of congenital vascular anomaly. Single stage unilateral craniotomy is recommended for surgical treatment.
\end{abstract}

Key words: aneurysm, distal anterior cerebral artery, middle cerebral artery, mirror image

\section{Introduction}

Distal anterior cerebral artery (ACA) aneurysms account for approximately $2 \%$ to $5.5 \%$ of all intracranial aneurysms. ${ }^{4,9,11,13)}$ Multiple aneurysms of the distal ACA are present in as many as $55 \%$ of cases. $^{2,7,13)}$ However, distal ACA mirror aneurysms are extremely rare. We treated a patient with four aneurysms, two mirror aneurysms located on the bilateral distal ACAs and two aneurysms on the right middle cerebral artery (MCA).

\section{Case Report}

A 52-year-old woman was referred to our department from another institute on the 2nd day after subarachnoid hemorrhage (SAH). She was awake and oriented with SAH of World Federation of Neurological Surgeons scale grade I. Cranial computed tomography (CT) revealed diffuse SAH in the interhemispheric fissure and bilateral sylvian fissures. Magnetic resonance (MR) imaging showed diffuse hemorrhage and a mass in the interhemispheric fissure of approximately $0.8 \mathrm{~cm}$ diameter, which was thought to be an aneurysm

Received November 14, 2005; Accepted April 16, 2006

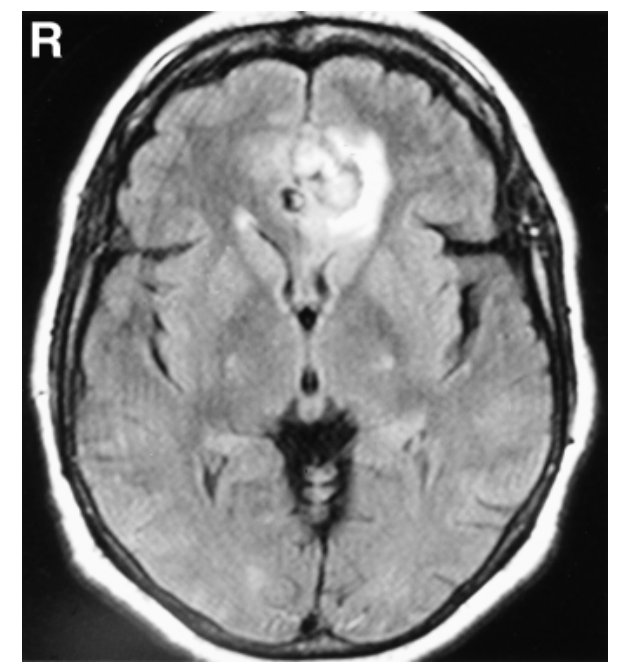

Fig. 1 Cranial axial $\mathbf{T}_{1}$-weighted magnetic resonance image revealing a mass lesion with surrounding cerebral edema in the left frontal lobe and probable aneurysm formation in the interhemispheric space.

(Fig. 1). Digital subtraction angiography (DSA) demonstrated two aneurysms on the bilateral pericallosal arteries (PCAs) at the same location and two right MCA aneurysms (Fig. 2). The bleeding pattern on cranial MR imaging clearly indicated 


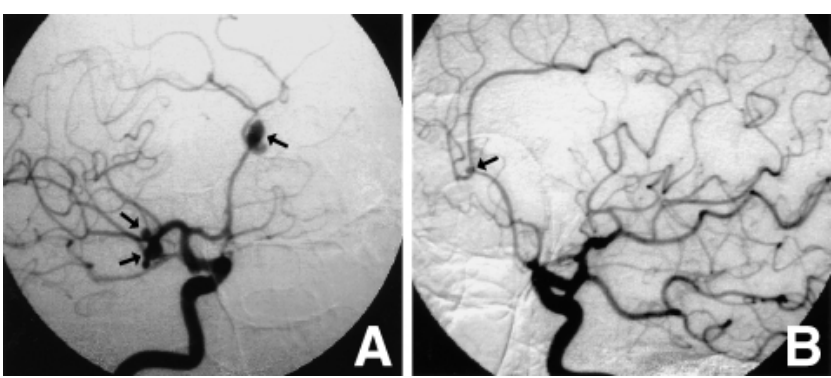

Fig. 2 A: Right carotid angiogram, anteroposterior view, demonstrating a 9-mm diameter aneurysm at the right pericallosal artery (PCA)-callosomarginal artery (CMA) bifurcation and two right middle cerebral artery aneurysms (arrows). B: Left carotid angiogram showing a 2-mm diameter aneurysm at the left PCA-CMA bifurcation (arrow).

bleeding from a distal ACA aneurysm, but we could not decide on which side.

Two craniotomies, a right interhemispheric and a right pterional craniotomy with bifrontal skin incision, were performed in the same session on the 4th day after SAH. The right distal ACA aneurysm was found to be intact, but thrombosed and adhered to the cingulate gyrus, so we could not explore and clip this aneurysm. Therefore, the left distal ACA aneurysm was the ruptured aneurysm. This aneurysm was clipped without difficulty. The two aneurysms on the right MCA were also clipped successfully.

The postoperative period was uneventful. The right distal ACA aneurysm could not be embolized because of thrombosis in the postoperative period. Postoperative DSA showed successful clipping of the other aneurysms. Follow up of the patient continues because of the thrombosed right distal ACA aneurysm (Fig. 3).

\section{Discussion}

Bilateral distal ACA aneurysms in mirror locations are extremely rare. ${ }^{5,6,10)}$ Twenty-three patients had 10 bilateral distal ACA aneurysms in one series, but only three of the 10 were clipped, as the other aneurysms were too small, so were coagulated and covered with muscle. ${ }^{13)}$ Three of 14 cases $^{4)}$ and two of 20 cases $^{12)}$ had bilateral distal ACA aneurysms in mirror positions. The tendency of multiple distal ACA aneurysms to occur is well known, but in the present case two additional MCA aneurysms were present in the absence of congenital vascular anomalies.

Distal ACA aneurysms are frequently associated



Fig. 3 Control digital subtraction angiograms, right anteroposterior view (A) revealing the thrombosed right distal anterior cerebral artery aneurysm and no residual filling of the right middle cerebral artery aneurysms, and left lateral view (B) showing no residual filling of the left pericallosal artery aneurysm.

with congenital anomalies. ${ }^{1)}$ Anatomic variations in the distal ACA occur in $25 \%$ of cases. ${ }^{1)}$ An example is a case of bridging artery at the bifurcation into the PCA and callosomarginal artery (CMA) called the "supreme" anterior communicating artery. ${ }^{4)}$ The flow disturbance caused by such a developmental anomaly might also account for mirror aneurysms. However, one previous patient with bilateral distal ACA mirror aneurysms had no vascular anomaly. ${ }^{10)}$

Only a few cases of large and giant distal ACA aneurysms have been reported..$^{2,7,8)}$ Distal ACA aneurysm tends to rupture before any mass effect manifests. ${ }^{7,8)}$ In one series, $67 \%$ of the ruptured distal ACA aneurysms were less than $5 \mathrm{~mm}$ in diameter. ${ }^{7)}$ Therefore, most patients with distal ACA aneurysms present with SAH. Intracerebral hematoma in the frontal lobe is also common. ${ }^{3)}$ Hematoma formation may occur on the contralateral side to unilateral aneurysms. ${ }^{3)}$

DSA is the gold standard for the detection of vascular abnormalities. However, MR angiography demonstrated a case of bilateral distal ACA aneurysms. ${ }^{5}$ Conventional angiography revealed an aneurysm on the PCA-CMA bifurcation of the left or right distal ACA. Three-dimensional time-of-flight MR angiography then demonstrated two mirror aneurysms on the bilateral bifurcations. Therefore, preoperative MR angiography may be useful in patients with ruptured aneurysms.

Surgical treatment of distal ACA aneurysm presents various difficulties, including the narrowness of the interhemispheric fissure and the callosal cistern, the possibility of dense adherences between the cingulate gyri, the difficulty in establishing proximal control, and the high frequency of broad- 
based and sclerotic aneurysms in this location, particularly those involving the origin of the branching arteries and premature rupture. ${ }^{13)}$ Therefore, possible multiplicity of the aneurysms is important to investigate preoperatively. Distal ACA aneurysms are usually treated through an interhemispheric approach. ${ }^{13)}$

We could not decide which distal ACA aneurysm had bled preoperatively in our patient based on the CT and MR imaging findings, and two additional aneurysms were present on the right MCA. Therefore, we performed right pterional and right interhemispheric craniotomies in the same session. During the surgery, the left distal ACA aneurysm was identified as ruptured and was clipped successfully. However, the right distal ACA aneurysm had a broad base, and was thrombosed, calcified, and densely adhered to the ipsilateral cingulate gyrus, so we could not clip this aneurysm. This aneurysm also could not be embolized because of thrombosis in the postoperative period.

The most important factor affecting the mortality and morbidity is the presence of associated aneurysms. ${ }^{2)}$ Two stage surgery at two different sessions has been recommended for multiple aneurysms to reduce the mortality. ${ }^{2)}$ However, one stage surgery is also suggested, ${ }^{13)}$ with which we agree.

All patients with distal ACA aneurysm should be carefully evaluated with DSA for the presence of additional aneurysms due to the tendency for multiplicity. We recommend one stage surgery for these aneurysms. Intracerebral mirror aneurysms are rare on the distal ACAs, and can be approached through unilateral craniotomy.

\section{References}

1) Baptista AG: Studies on the arteries of the brain, II: The anterior cerebral artery: Some anatomic features and their clinical implications. Neurology 13: 825-835, 1963

2) De Sousa AA, Dantas LRD, Cardoso GTC, Costa BS: Distal anterior cerebral artery aneurysms. Surg Neurol 52: 128-136, 1999
3) Hamilton JG, Falconer MA: Immediate and late results of surgery in cases of saccular intracranial aneurysms. J Neurosurg 16: 514-541, 1959

4) Laitinen L, Snellman A: Aneurysms of the pericallosal artery: a study of 14 cases verified angiographically and treated mainly by direct surgical attack. J Neurosurg 17: 447-458, 1960

5) Mori T, Fujimoto M, Shimada K, Shin H, Sakakibara T, Yamaki T: Kissing aneurysms of distal anterior cerebral arteries demonstrated by magnetic resonance angiography. Surg Neurol 43: 497-499, 1995

6) Niijima KH, Yonekawa Y, Kawano T: [Bilateral pericallosal artery aneurysms in a mirror position]. No Shinkei Geka 17: 779-781, 1989 (Jpn, with Eng abstract)

7) Ohno K, Monma S, Suzuki R, Masaoka H, Matsushima Y, Hirakawa K: Saccular aneurysms of the distal anterior cerebral artery. Neurosurgery 27: 907-913, 1990

8) Sindou M, Guyotot P, Mertens P, Keravel Y, Athayde A: Pericallosal aneurysms. Surg Neurol 30: 434-440, 1988

9) Snyckers FD, Drake CG: Aneurysms of the distal anterior cerebral artery. A report on 24 verified cases. S Afr Med J 47: 1787-1791, 1973

10) Sousa J, Iyer V, Roberts G: 'Mirror image' distal anterior cerebral artery aneurysms. A case report of two patients with review of literature. Acta Neurochir (Wien) 144: 933-935, 2002

11) Sugar O, Tinsley M: Aneurysms of the terminal portion of the anterior cerebral artery. Arch Neurol Psychiatry 60: 81-85, 1948

12) Wishoff JH, Flamm ES: Aneurysms of the distal anterior cerebral artery and associated vascular anomalies. Neurosurgery 20: 735-741, 1987

13) Yasargil MG: Surgery of the Intracranial Aneurysms and Results. Microneurosurgery, vol II, Clinical Considerations. Stuttgart, George Thieme, 1984, pp 224-231

Address reprint requests to: C. Dinc, M.D., Icerenkoy Emniyet Cikmazi Sk. Aydin Kardesler Apt. No:4/16, 34752 Kadikoy/Istanbul/Turkiye.

e-mail:drcemdinc@yahoo.com 